\title{
Ways to Determine the Structural Resource of Highly Maneuverable Aircraft
}

\author{
Nicolae-Alin Niculescu ${ }^{1}$, Jenica-Ileana Corcău ${ }^{2}$, Nicuşor Cătălin Stancu ${ }^{1}$ \\ ${ }^{1}$ Research, Innovation and Flight Test Center Military Equipment and Technologies Research Agency \\ Craiova, Romania, niculescu.alin88@gmail.com, nicusorcatalinstancu@yahoo.com \\ ${ }^{2}$ Department of Electrical, Energetic and Aerospace Engineering, University of Craiova, Craiova, Romania \\ jcorcau@elth.ucv.ro
}

DOI: 10.52846/AUCEE.2021.1.12

\begin{abstract}
This paper emphasizes the importance of monitoring aircraft structural fatigue and proposes as paramount the analysis of military aircraft structural constitutive materials aging process, during flight. Aircraft structural health monitoring is defined as an evaluation process for the integrity of key structural components with the sole purpose of enhancing flight safety and mitigating maintenance risks and loses. Nowadays, aircraft are predominantly built from composite materials with at least two layers with different mechanical properties, which combined, result in a new composite material with highly superior resilience and elastic properties. Even though there are great advantages of using composite materials, when it comes to operating military aircraft, a mere visual inspection cannot be sufficient for observing or identifying structural damages within the composite layers of the aircraft. The focus of this study is on improving the process of monitoring the structural condition of highly maneuverable military jet aircraft by using the strain gauge marks methods. Monitoring aircraft structural condition can be easily implemented, both during maintenance and flight missions, by using a wide array of sensors that can generate specific sets of instructions for all processes involved in enhancing flight and maintenance safety. The main concern of implementing a structural evaluation, within aircraft maintenance processes, sits on preventing and not ameliorating incidents and accidents during flight. As a result of the continuous research and testing, it has been proved that the operational resource of the aircraft structure, provided by the manufacturer, can be easily reached in a lot lesser flight hours than initially anticipated, by engaging the aircraft in specific operational missions and, therefore, inflicting pronounced fatigue on the aircraft structure.
\end{abstract}

Cuvinte cheie: monitorizarea aeronavei, structural, metoda mărcilor.

Keywords: monitoring aircraft, structural, marks method.

\section{EVOLUTION OF AIRCRAFT BUILDING AND MAINTENANCE PROCESS}

Aircraft building, in the last decade, has known a significant improvement, from both navigation and flight control systems and the material used for structure building stand points. All these technological advances have had the main purpose of increasing the flight safety level and, therefore, statistically, airborne transportation has become, nowadays, the safest and the most reliant way of reaching different locations.
Composite materials offer a series of advantages in comparison with the conventional materials as fatigue resilience, corrosion resilience, advantageous specific resistance and resilience (according to weight ratio). The most important aspect regarding composite materials is that its chemical and mechanical proprieties can be altered in order to reach maximum resilience for the known types and directions of strains within the aircraft structure [1].

Although composite materials come with lots of upsides, whether it is low weight, superior chemical and mechanical proprieties or facile structure building, it is important to mention that the composite layers present a great risk in having trouble to timely identify structural deteriorations.

The most important deteriorations that can occur within the composite structure are: fibre detachment from its matrix, cracks in the matrix, gap expansions within the matrix, delamination and fibre breakings; these alterations are, actually, stages within the progressive cracking process of the composite structures. The main concern is that, in the case of composite structures, a mere visual inspection does not qualify as a tool for identifying structural deteriorations and, consequently, using composite materials in the aviation industry could represent a safety issue [11].

Shifting the paradigm in what regards the environmental preservation is yet another reason for which composite materials are prone to be used in the aviation industry. ACARE (Advisory Council for Aviation Research and innovation, Europe), has set environmental goals as reducing carbon dioxide $(\mathrm{CO} 2)$ emissions by $50 \%$, reducing acoustic emissions by $50 \%$ and reducing oxide nitrate by $80 \%$ [2].

Even though, by using composite materials, the cost of developing and certifying a new type of technology for aircraft maintenance and aircraft structural monitoring has considerably risen, the benefits are deducted from the time reduction for the aircraft maintenance process and from decreasing the added risks of aviation accidents. It can be estimated that by implementing a method of continuously monitoring the aircraft structural condition, even though it increases the costs of aircraft maintenance processes by $20 \%$, the affected time for aircraft maintenance will drop by $40 \%$ - which is the main concern and cost generator for an airline company [3].

Since the first time composite materials were used in the aviation industry, in 1957, the interest in these materials has met a great expansion, mostly in the military aviation industry. The use of these materials has increased 
not only due to the obvious advantages as mass reduction, high resilience or increased resistance, but also because of the continuous maturing process of this technology and its applications.

An annual report of Lufthansa has recorded a number of 1648 events on a fleet of 243 monitored aircraft and the statistics showed that the average repair costs reached 33 million euros per year. The average repair time was 3.5 days which led to a loss of 200.000 euros for airplane in the fleet. Since 1996 a special form has been implemented in order to record maintenance problems and between 1999 and 2007, a number of 104 relevant ground incidents have been studied [12].
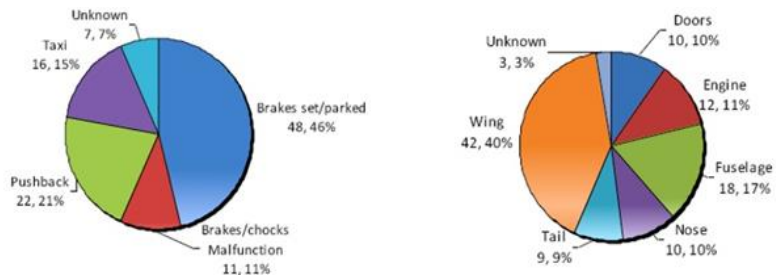

Chart 1. Position of aircraft during incident

\section{Chart 2.Damaged} structural area

The survey specified that most incidents were produced due to ground operations and by the impact with ground vehicles, the wing being the area with the highest probability of being damaged [12].

Due to these reasons, aircraft structural condition monitoring becomes a primordial necessity and of utmost importance in the aircraft maintenance field, both civilian and military, this being the first step for increasing the safe use of aircraft and increasing aircraft availability, by reducing time and costs for aircraft maintenance and repair [4].

\section{DIFFERENT METHODS OF MONITORING THE STRUCTURAL CONDITION}

The most important step in the full analysis of structural damages is their identification. There are many techniques of detecting structural damages and the costs rise in accordance with the technology used for the process.

The most widespread and also the cheapest one is visual inspection through which damages can be discovered by observing different surface deformations or paint cracks.

The second method is called the tap-test or coin-test and it consists of using special electronic hammers having an accelerometer at one end that can measure the response time between impulses. An internal structural damage will reduce the structural rigidity and the transmitted signal will have lower amplitude and bigger response time interval [16].

Other way of identifying propose the use of thermal images based on emitting energy waves (ultrasounds, microwaves, UV) towards the surface of the structure. The periodic waves penetrate the fabric of the structure, where they are absorbed, until a discontinuity is met and then the waves are partially reflected backwards. The

reflected wave interferes with the initially emitted wave, thus, generating a thermic pattern over the local surface. The internal structure can finally be analysed by observing the thermic patterns across the airplane surface.

A different and more expensive direction proposed for monitoring the structural condition is the use of repairing technologies, which, in the case of composite materials, have not reached at full maturity in what concerns their development and, therefore, they were not unanimously accepted. Repairing technologies suggest the use of different materials imbedded within the structure of the aircraft that can be activated to repair small deformations, cracks or damages, even during flight [15].

Structural Health Monitoring is the name of most widespread safety programme used by the aircraft users and manufacturers for the maintenance process. Structural Health Management is defined as an evaluation of key structural elements` integrity with the sole purpose of mitigating risks and reducing losses of any kind. There are two approaches for monitoring structural integrity: degradation monitoring and operational monitoring.

Degradation monitoring can be obtained through more direct methods such as evaluating the fatigue level of the structure, observing a relation between damages and implications of weather elements and identifying damages rendered by accidents and mechanic shocks.

Operational monitoring consists of using indirect ways of measuring the structural fatigue with the help of sensors mounted on the soft spots of aircraft. Operational monitoring is a continuous process of evaluating and preventing serious structural damages and deformations in accordance with data available. The data used for calculating and analysing the structural fatigue can be time since last repair, the strain forces applied to the structure during flight, temperature during flight and other operational data.

The costs of developing new repairing process and maintenance technology are very high but the benefits surpass them. It is estimated that, by implementing such a technology on aircraft, can rise the maintenance costs by $20 \%$ but could also reduce inspection time intervals by $40 \%$, which is the main source of money drain for an airline operator.

It can be stated that implementing a structural monitoring technology is a necessity if the goal is to reduce the stationing time of airplanes and mitigate safety risks during flight.

\section{STRAIN GAUGE MARKS METHOD}

Strain gauge marks represent the most widespread type of sensors used for monitoring structural condition as it presents great advantages. They both are used in the analysis of aircraft structure, within the aviation incidents, that result in structural damages of external causality and in monitoring the structural fatigue during flight maneuvers, by measuring the strain resistive voltage effect. The strain resistive voltage effect represents the resistive proprieties modification of a conductor by applying a strain effort that determines a certain elongation or suppression. The strain resistive voltage effect has been more widely used after roughly 75 years 
since it was discovered, in 1856, by Lord Kelvin, once the first strain gauge marks started being used.

Since then, strain gauge marks have known a rapid development, due to both its constructive simplicity and the relative simplicity of converting the resistive voltage variation into usable signal (the circuit is usually a Wheatstone bridge) [5].

\section{Operating principle}

The force and moment convertors are mainly useful for monitoring dynamic structures which are subject to variable strain loading regimes (e.g. tool apparatus, robots, conveyor gauges, etc.); in these situations, the strain takes the form of a force vector and determining its direction is the most important step for completing the structural deformation calculus.

A particular case in which only the absolute value of the force vector is important, vector direction not being important, is the action of calculating the weight of a mass. In this case the value of the force vector can be determined by using the acceleration of the dynamic structure:

$$
F=k m a
$$

Where $F$ is the force vector that presses on the mass $m, a$ represents the acceleration, and $k$ represents a coefficient dependent of the measuring unit. The IS for $[\mathrm{m}]=1 \mathrm{~kg}$ and $[a]=1 \mathrm{~m} / \mathrm{s}^{2}, k=1$ and $[F]=1 \mathrm{~N}$.

The torque $M$ is generated by the relation between the vector and the distance between the vector direction and the rotation (center) axis:

$$
M=F l \text { or } M=J a_{u}
$$

where $l$ is the lever arm, $J$-inertia moment, $a_{u}$ - angular acceleration; the torque could take the form of bending, torsion or sectioning; the usual measurement that takes place as a part of the industrial processes is measuring torsion moments.

The IS measuring unit for torque is [N m]. Strongly connected to measuring the stretch and compression forces is the calculus of the relative elongation (measured through the unit effort), which represents the deformation rendered by the force acting on the surface unit within a solid form:

$$
\varepsilon=\sigma / E
$$

where: $\varepsilon$ is the deformation; $\sigma$ - unit effort; $E$ - elasticity module. Usually $\varepsilon$ measuring unit is expressed as $[\mathrm{mm} / \mathrm{m}]$ or $[\mu \mathrm{m} / \mathrm{m}]$.

Considering a fixed conductor with a section $A$, length $l$ and resistivity $\rho$, its resistivity variation due to dimension variation produced by the $\Delta l$ elongation is going to be:

$$
\Delta R=(\rho / A) \Delta \mathrm{l}-\rho\left(1 / A^{2}\right) \Delta A+(1 / A) \Delta \rho
$$

And by dividing the relation to $\mathrm{R}$, the relative variation is going to be:

$$
\Delta R / R=(\Delta l / l)-(1 / l) *(\Delta A / A)+(1 / l)^{*}(\Delta \rho / \rho)
$$

$$
\Delta A / A=-2 \mu(\Delta l / l)
$$

where $\mu$ represents the Poisson coefficient (the division of transversal contraction by its elongation); it can be determined, for its resistivity, a linear variation with the volume $V$, having the form:

$$
\Delta \rho / \rho=\mathrm{k}(\Delta V / V)=k(\Delta l A+l \Delta A) / V=k(1-2 \mu)(\Delta l / l)
$$

Results the expression:

$$
\Delta R / R=(\Delta l / l)[1-2 \mu+k(1-2 \mu)]=K(\Delta l / l)=K_{\varepsilon}
$$

In practice the strain resistive voltage elements are known as strain gauge marks, where the $\mathrm{K}$ coefficient from the last relation is called the mark factor. It is dependent on the nature of the material (k coefficient) and on the production technology of the mark, representing the sensibility of the sensor (the relation between the relative variation of its resistance and the relative elongation).

\section{CASE Study: Structural Condition ANALYSIS OF THE IAR-99 AIRCRAFT}

Structural condition monitoring of aircraft, during a life cycle, represents the most important step in determining the real structural resource consumption with the purpose of:

- Increasing safety within the flying activities;

- Reconsidering the aircraft technical resource;

- Updating the maintenance technical documentation;

- Maintenance cost reduction during the aircraft life span;

This paper proposes to use the in-flight practical testing and evaluating in order to validate the theoretical model used for monitoring the structural condition of the IAR-99 aircraft, equipped with strain gauge marks. The evaluation of IAR-99 aircraft structural condition will be done by using the data recorded by the SAIMS on-board recording system, counting the loading cycles according to the Gstrain levels and finally comparing results between the data collected from both strain gauge marks and SAIMS on-board recording system.

Based on the results of the analysis and centralizing the real aircraft strain loading data, important decisions can be made in what regards the maintenance process of the entire fleet of aircraft. The purpose is to monitor the fatigue condition of the aircraft, based on the mission profiles and according to the loading spectre, determined by measuring structural linear specific deformations of the structure and flight parameters. Using data from the inflight testing, estimations can be made for the structural components' life span, by determining the tensions resulted from the specific linear deformations, provided by the strain gauge marks and by calibrating the theoretical calculus model of the finite structural elements specified by the manufacturer [6].

Since relative variation of its area can be expressed as: 


\section{General testing conception}

Calculating the aircraft life span is being made by taking into consideration different subparts of the structure, by facilitating the initial building process of the aircraft. Data analysis, after in-flight testing, allows the comparison of recorded data with the initial fabrication data of the aircraft. A number of 12 specific areas of the aircraft have been chosen as representative for monitoring the structural fatigue condition. In figure 1 it is represented the IAR-99 aircraft and the specific areas in which strain gauge marks have been placed.

The areas on which strain gauge marks have been mounted next to high tensions zones and are specific for calculating the real structural life span of the aircraft. According with the studies conducted by the manufacturer, the areas with the maximum strain pressure are the ones where the wings meet the main body of the aircraft. In-flight testing and creating a relation between deformation and resistive tensions lead to calculating the theoretical model for the aircraft structure and updating the real life span resource of the aircraft structure [7]. This latter calculus should not be very different from its original theoretical model because there have been applied safety coefficients and, thus, covering the strain loading factors for the aircraft.

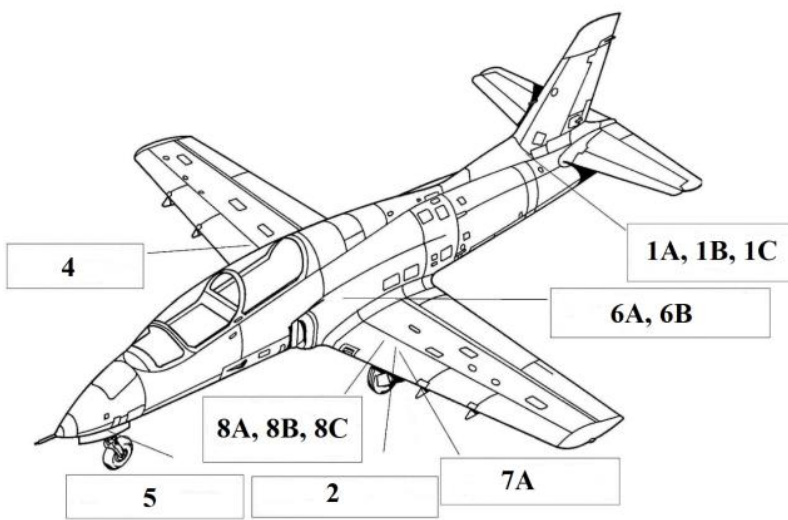

Fig.1. Areas where strain gauge marks have been placed.

Technical data representative for mounting the strain gauge marks:

- the strain gauge marks have been mounted near the maximum tension areas and, therefore, only after the theoretical model had been calibrated, the real life span calculus could be made, according to specific deformation data recorded from the flight testing.

- the strain gauge marks mounted on the aircraft have a correct indication area between $-10^{\circ} \mathrm{C} \ldots+45^{\circ} \mathrm{C}$, reason for which the flight testing had to be made under temperature restrictions.

establishing the loading/unloading strain model during flight testing led to an asymmetric alternant cycle where $S$ max $>|S \min |$ and to an asymmetric coefficient (tension relation $R=S \min / S \max$ ) for the negative cycle.

- the surfaces susceptible for fatigue breakings are the bolted structural elements, surfaces with screws and bolts, these being areas with high strain tension that can transmit the axial cutting forces to the bolting elements.

The testing and evaluation activities have been conducted through the means of recording a big amount of flight data and creating a sufficient database to show the repeatability of the correct results. Altogether, different tension values can be set for the strain gauge marks areas in accordance to the acceleration levels 5 different types of validation sorties have been conducted, which led to verifying data rendered by a 2 year period of flight training with the same aircraft. Model testing, implemented on the analysis ground station for monitoring the structural condition of IAR-99 aircraft, has been made through recording parameters by SAIMS system for all sorties and creating a database for the measurement of fatigue loading cycles.

\section{V.Testing ACTIVITIES}

The general testing and evaluation concept of producing the theoretical model used for monitoring IAR99 aircraft structure, equipped with strain gauge marks, is structured as follows.:

- $\quad$ Stage 1: - Executing flight testing for the purpose of extracting data from strain gauge marks;

- $\quad$ Stage 2: - Extracting data rendered by the strain gauge marks and creating a database with the newly available data;

- $\quad$ Stage 3: - Analysing the database and creating a function (relation) between the specific linear deformations and the normal $\mathrm{Nz}$ accelerations;

$\bullet$

Stage 4: - Executing flight testing in order to validate the function (relation);

- $\quad$ Stage 5: - Gathering the strain loading cycles data in accordance to the normal $\mathrm{Nz}$ acceleration for the flight sorties that used the SAIMS system.

In order to confirm results from previous sorties and structural condition data correctness, the specific function validation has been obtained through specialized flight procedures and specific manoeuvres, predominantly used for 5 types of profiles specific for military missions:
1)
2)
3)
Stable maneouvers and Navigation;
Air-to-air attacks;
Air-to-ground attacks;
Aerobatic manoeuvres;

Flight testing sorties have been done between heights of $200 \mathrm{~m} \div 5000 \mathrm{~m}$, clean profile of aircraft, with a crew of 2 pilots, take-off weight of $4910 \mathrm{~kg}$, total fuel quantity of $1300 \mathrm{l}$ and play-time between $10 \mathrm{~min}-45 \mathrm{~min}$.

In order not to apply any correction, the temperature interval, during all 5 types of sorties, has been kept within the strain gauge marks temperature restrictions $-10^{\circ} \mathrm{C} \ldots$ $+45^{\circ} \mathrm{C}$, at any given altitude.

Using strain gauge marks, different strain tensions have been recorded for every flight manoeuver, in correspondence with the specific strain loading cycles, suffered by the IAR-99 aircraft, used for flight tests. Thus, a relation between different types of flight missions and the equivalent strain loading cycles has been established, which can be very useful in determining the real structural fatigue level for each flight manoeuver.

It has been concluded that, mostly, the resulted strain tensions are within the limitations provided by the manufacturer, actually being lower in the case of calculated limits for elastic fatigue. It can be observed that the recorded values, transformed into strain tensions, are 
very close to the ones provided by the manufacturer as technical resource for IAR-99 aircraft structure.

Next, a database has been created using the results recorded by the SAIMS system. The analysis of this database can lead to obtaining a theoretical model to correlate the strain loading cycles and normal G-strain acceleration levels. The purpose is to measure and analyse strain loading cycles for every aircraft in the fleet and impose safety measures when the technical resource provided by the manufacturer is depleted, in order to anticipate the right time to conduct more intricate and complex structural verification and maintenance [14]. Composite materials represent a much bigger part in the building process of structural components and elements of aircraft. Although the cost is bigger than the conventional materials, aeronautical industry has appreciated composite materials' mechanical proprieties as being the best solution for building airplanes and also reducing weight. Introducing composite materials in the aviation industry comes with the burden of developing new types of maintenance technology that can also be applied to isotropic materials [8].

Knowing the way materials behave is paramount for anticipating its response to different types of strains in order to considerably reduce the maintenance costs. A crack within the aircraft structure does not necessarily mean that the structure is damaged beyond repair. Continuously monitoring the structure allows further using of damaged components until the anticipation of the right time to change it, by predicting the critical length of the crack. Identifying the deformation of a structure is fundamental. Materials, mostly the composite ones, can present internal deteriorations that cannot be observed with the naked eye. Even though there is multiple detection technics, the visual inspection is still the most widespread. Only when more dangerous deformations are identified, more precise, but more expensive detection technics are applied [9].

New materials rely on new technics for exploiting them, characterized by high costs. Starting with the main idea for which the use of composite materials has been adopted, reducing costs on the long run, it is considered mandatory to implement new technologies of using these new materials in order to efficiently apply the entire industrial process, from both economical and operational stand points.

By using the data recorded during a 2 years period and for an amount of, roughly, 300 flight hours, with the help of the SAIMS system, a database has been created and the available data for comparison are presented in table 1.

Therefore, by comparing strain loading cycles, provided by the manufacturer as technical resource, with the strain loading cycles resulted from the relation between data recorded by SAIMS system and data taken from the strain gauge marks, a correct fatigue level of the aircraft structure can be established, for each aircraft, according to its flight history.

It can be observed that within 300 real flight hours, the aircraft has already consumed its technical resource provided by the manufacturer for 1000 theoretical flight hour, according to the strain loading/unloading cycles:
TABLE 1. STRAIN LOADING CYCLE COMPARISON

\begin{tabular}{|c|c|c|c|c|c|}
\hline 1 & 2 & 3 & 4 & 5 & 6 \\
\hline $\begin{array}{l}\text { G- } \\
\text { factor } \\
\text { level }\end{array}$ & $\begin{array}{l}\text { Strain } \\
\text { loading } \\
\text { cycles } \\
\text { provided } \\
\text { by } \\
\text { manufactu } \\
\text { rer (per } \\
1000 \\
\text { flight } \\
\text { hours } \\
\text { resource) } \\
\text { (2) }\end{array}$ & $\begin{array}{l}\text { Number } \\
\text { of } \\
\text { strain } \\
\text { loading } \\
\text { cycles } \\
\text { for } 300 \\
\text { flight } \\
\text { hours } \\
\text { (SAIM } \\
\text { S) } \\
\text { (3) }\end{array}$ & $\begin{array}{l}\text { Number of } \\
\text { real strain } \\
\text { loading } \\
\text { cycles } \\
\text { available } \\
\text { after } 300 \\
\text { flight hours }\end{array}$ & $\begin{array}{l}\text { Theoretical } \\
\text { number of } \\
\text { real strain } \\
\text { loading } \\
\text { cycles for } \\
3000 \text { flight } \\
\text { hours }\end{array}$ & $\begin{array}{l}\text { Theoretica } \\
1 \text { number } \\
\text { of real } \\
\text { strain } \\
\text { loading } \\
\text { cycles } \\
\text { available } \\
\text { after } 3000 \\
\text { flight } \\
\text { hours } \\
(6)=(2) \times 3- \\
(5)\end{array}$ \\
\hline 2,45 & 17.000 & 4769 & 12.231 & 47690 & 3310 \\
\hline 3,15 & 9.500 & 4021 & 5.479 & 40210 & -11710 \\
\hline 3,85 & 6.500 & 2594 & 3.906 & 25940 & -6440 \\
\hline 4,55 & 4.500 & 1263 & 3.237 & 12630 & 870 \\
\hline 5,25 & 2.500 & 458 & 2.042 & 4580 & 2920 \\
\hline 5,95 & 1.500 & 54 & 1.446 & 540 & 3960 \\
\hline 6,65 & 300 & 5 & 295 & 50 & 850 \\
\hline 7,35 & 150 & 1 & 149 & 10 & 440 \\
\hline 0 & 500 & 1343 & -843 & 13430 & -11930 \\
\hline$-0,36$ & 200 & 958 & -758 & 9580 & -8980 \\
\hline$-0,72$ & 100 & 489 & -389 & 4890 & -4590 \\
\hline$-1,08$ & 60 & 235 & -175 & 2350 & -2170 \\
\hline$-1,44$ & 35 & 86 & -51 & 860 & -755 \\
\hline$-1,8$ & 30 & 34 & -4 & 340 & -250 \\
\hline$-2,16$ & 25 & 12 & 13 & 120 & -45 \\
\hline$-2,52$ & 20 & 4 & 16 & 40 & 20 \\
\hline$-2,88$ & 15 & 1 & 14 & 10 & 35 \\
\hline
\end{tabular}

- positive cycles for $2,45 \mathrm{~N} \mathrm{z}-28,05 \%$;

- positive cycles for $3,15 \mathrm{~N} \mathrm{z}-42,32 \%$

- positive cycles for $3,85 \mathrm{~N} \mathrm{z}-39,90 \%$;

- positive cycles for $4,55 \mathrm{~N} \mathrm{z}-28,06 \%$;

- positive cycles for $5,25 \mathrm{~N} \mathrm{z}-18,32 \%$;

- positive cycles for $5,95 \mathrm{~N} \mathrm{z}-3,6 \%$;

- positive cycles for $6,65 \mathrm{~N} \mathrm{z}-1,66 \%$;

- positive cycles for $7,35 \mathrm{~N} \mathrm{z}-0,66 \%$.

(Column 3 cycles as compared to Column 2 cycles)

Regarding the negative cycles specter, the resource given for 1000 theoretical flight hours has been completely depleted in just 300 real flight hours, for GForce values between 0 and $-1,8 \mathrm{~g}$. Thus, the number of strain loading cycles is bigger than it was initially calculated for 3000 theoretical flight hours. As a side note, the low number of negative strain loading cycles provided initially by the manufacturer has been calculated this way in order to keep the aircraft weight as low as possible.

The highlighted red-colored loading cycles, in table 1 , represent the number of cycles that are not covered by the theoretical cycles/resource provided by the manufacturer.

Data show that for the G-force intervals of $(+3,15 \ldots$ $+3,85)$ and $(0 \ldots-2,16)$, the real loading cycles are bigger than the ones provided by the manufacturer. Therefore, more thorough and more often investigations are required, in order to prevent in-flight safety issues.

It is obvious that the theoretical resource provided by the producer is to be met way before it was foreseen. Therefore, by utilizing the proposed method of monitoring the structural condition, the real remaining technical resource can be determined for each airplane, according to 
the way it was used, and also it would increase the safety level for both flight procedures and maintenance processes.

At the end of this analysis a set of rules could emerge in what concerns the flight missions and specific use, for every airplane, by calculating the remaining number of strain loading cycles, taking into consideration data recorded from all previous sorties.

A new emerging direction is represented by the use of intelligent materials with self-repairing capabilities. Although this concept is very interesting, it is not mature enough in order to be adopted by big aviation manufacturers. Another problem identified for this type of materials is the high production costs. There are numerous efforts from civilian airliners to reduce costs with stationed airplanes due to maintenance or incident-related activities. The development of a voluntary system using guidance from anonymous declarations regarding incidents, implemented by the American Agency ASRS, has allowed the reduction of incident on the ground, mainly by improving all operational and maintenance procedures. A system of sensors, for monitoring the structural condition of an aircraft, would reduce stationing time periods, affected by mandatory inspections and, thus, reduce general costs of operating any type of aircraft. [10].

The problems of the newly proposed maintenance technology are connected to limitations given by the implementation and certification of the new monitoring system. The next step into this area is to include the structural monitoring system in the initial processes of airplane building. Flight data have highlighted the possibility of directly monitoring the structural strains of an aircraft with implications in identifying the difference between the real technical resource and the theoretical resource specified by the manufacturer.

Monitoring the structural condition allows the current fatigue state of the tested aircraft and, by interpolating data, it can be determined how the entire fleet is currently affected. This leads to the optimization of maintenance planning and processes for all airplanes in the fleet. Direct structural monitoring and the determination of the remaining technical resource cannot replace planned maintenance specified by the manufacturer but can represent the basis for future safety recommendations and measures for improving the general use of airplanes. The results of the recorded linear specific deformations can be compared with the theoretical calculus and it can offer important data for calibrating the theoretical model.

With the help of this model, a precise estimation of the remaining technical resource can be made and, by knowing the total flight hours of an aircraft, the structural fatigue condition can be determined.

The results obtained from the validation of the theoretical model of calculus, presented in this project, can be used in order to analyse the structural condition with no strain gauge marks mounted on. If the total flight history of the fleet is not available, an approximation can be made using the 5 types of flight missions, previously recorded, each type of mission corresponding to a certain number of strain loading cycles for every G-factor level obtained.

In this way, possible measures can be identified when theoretical technical resource, given by the manufacturer, is depleted before its time, as rescheduling the planned period for capital revision specified by the producer, respectively $800 \pm 50$ functioning hours or/and $8 \pm 0,5$ years for IAR-99 aircraft.

The process of monitoring the structural condition of aircraft is rapidly becoming a necessary method for determining technical resource for airplanes, thus, reducing safety problems, related to flight activities, and eliminating different technical errors. Most aircraft manufacturers, both military and civilian, are investing in different methods of anticipating technical problems in order to reduce maintenance cost and aeronautical safety incidents.

Of course, there are more efficient methods of monitoring and repairing aircraft than the one using strain gauge marks, as making use of intelligent materials ASRC type (Active Sensing Repair Composite), characterized by implementing of self-repairing materials while the structural damages are being produced. Although this advanced system could offer better results, it implies very big costs due to the fact that this technology hasn't yet been developed to be mature enough to offer a decent safety level for practical utilization of it [11].

There are two approaches regarding structural integrity monitoring: structural degradation monitoring and operational monitoring. In general, structural degradation monitoring can be made only after structural damages, as cracks, appear and are already visible. Operational monitoring implies evaluation of material fatigue level, extreme strains suffered by the material and effects of environmental factors on the structure, even before cracks or stress marks become visible [12].

In what concerns operational monitoring, the most efficient method, from cost and safety stand points, has been proved to be the one using strain gauge marks. The proposition of this method is applied mostly to military aircraft, used for flying in all types of missions.

\section{CONCLUSIONS}

Even though aviation is a very standardized area, the usage of military aircraft in all types of mission and pushing its structure to the limit, make it very hard to implement a standardization of the way we determine the real structural technical resource.

The main goal of this paper was to efficiently monitor the structural condition of a highly maneuverable aircraft using strain gauge marks and it has been demonstrated that the operational resource given by the manufacturer can easily be depleted within a smaller number of flight hours than initially anticipated, by executing a number of specific sorties, that can stress the aircraft structure way above the normal use of it. The calculated model for every type of mission can enhance the maintenance process by measuring the structural strain loading cycles and, consequently, the operational resource of aircraft structure, by producing a database containing information such as the types of sorties, play-time and the results recorded by the strain gauge marks.

The role of this study is to equip every aircraft with strain gauge marks, in order to improve structural condition monitoring or at least to use the calculated 
theoretical model. By utilizing the strain gauge marks method for monitoring the structure of highly maneuverable aircraft, a database can be created using strain loading/unloading cycles for every type of flight mission and, therefore, structural damages of any kind can be anticipated for every aircraft, according to its previous utilization, in different operational types of missions.

The theoretical model, validated through flight testing activities, corroborated with the database for every aircraft and type of mission, represent an effective way to reduce maintenance costs and improve aeronautical safety, by anticipating and preventing technical incidents or even aviation accidents.

Source of research funding in this article: Research, Innovation and Flight Test Center Military Equipment and Technologies Research Agency Craiova and Department of Electrical, Energetic and Aerospace Engineering financed by the University of Craiova.

Contribution of authors:

First author $-40 \%$

First coauthor $-30 \%$

Second coauthor $-30 \%$

Received on July 10, 2021

Editorial approval on October 25, 2021

\section{REFERENCES}

[1] E. F. Bhrun, B. S., M. S., C.E., "Analysis and Design of Flight Vehicle Structures" - , Tri-State Offset Co., 1973.

[2] F. Smith - Advisory Council for Aviation Research and Innovation, Avalon Consultancy Services Ltd, 2013.
[3] "Structural Health Monitoring System Implementation on Civil Aircraft", SMART MATERIALS, STRUCTURES \& NDT in AEROSPACE. Montreal, Quebec, Canada, 2011.

[4] Alwyn and J Topham - "Boeing Dreamliner Incidents Raise Safety Concerns", Aviation Week, 2013.

[5] RD3 WichiTech Industries. Digital Electronic Tap Hammer. Operations and Maintenance Manual;

[6] G. Georgeson, S. Lea, and J. Hansen, "Electronic Tap Hammer for Composite Damage Assessment", SPIE - The International Society for Optical Engineering, 1996.

[7] H. Kim, "Impact Damage Formation on Composite Aircraft Structures", JAMS 2014 Technical Review, 2014, March 25-26.

[8] H. Massengill, "Applied Aerodynamics" - Commercial Flight. Aerospace America, p. 7. 97, 2005

[9] S. Mancini, G. Tumino, and P. Gaudenzi, "Structural Health Monitoring for Future Space Vehicles", Journal of Intelligent Material Systems and Structures, 2005.

[10] M. Kaden \& R. Keck, "Repair Concept Supported by Laser Removal and Inductive Heating", The 28th Intern. Congress of the Aeronautical Sciences. Brisbane, Australia, 2012.

[11] G. Marsh, "The challenge of composite fuselage repair", Reinforced Plastics, 2012

[12] B.G. Kanki and C.L. Brasil, "Analysis of Ramp Damage Incidents and Implications for Future Composite Aircraft Structure", Proceedings of the 15th International Symposium on Aviation Psychology. Dayton, OH: Proceedings of the 15th International Symposium on Aviation Psychology, Dayton, OH, 2009.

[13] MILHDBK5H -Military Handbook - Metallic Materials and Elements for Aerospace Vehicle Structures, 1998.

[14] ASTM E 1049-85 - Standard Practices for Cycle Counting in Fatigue Analysis, 1997.

[15] E.F. Bhrun et al., "Analysis and Design of Flight Vehicle Structures", Tri-State Offset Co., 1973.

[16] Niculescu N.A., Corcau J., Gavril A. "Integrated System for Monitoring Aircraft Structural Condition by Using the Strain Gauge Marks Method", International Conference on Applied and Theoretical Electricity (ICATE) 2021, 27-28 May, IEEE conference; 10.1109/ICATE49685.2021.9465010; ISBN:978-17281-8036-6.

[17] RD3, WichiTech Industries. Digital Electronic Tap Hammer. Operations and Maintenance Manual. 